\title{
Transmutation Operator Method for Solving Heat Conduction Problem
}

\author{
Oleg Yaremko, ${ }^{1, *}$, Natalia Yaremko² \\ ${ }^{1}$ Moscow State Technological University "STANKIN ", RU-127055, Moscow, Russia \\ ${ }^{2}$ Penza State University, RU-440026, Penza, Russia
}

\begin{abstract}
The transmutation operator method is extended to the case of functions of two variables. The transmutation operator flattens the function, i.e. the transmutation operator replaces a function with discontinuous partial derivatives on the coordinate axes by a continuously differentiable function. The work reveal the properties of the transmutation operator, and prove the commutativity of the transmutation operator and the Laplace operator. It was found that the Cauchy problem for the Laplace equation with internal conjugations in an unbounded domain can be replaced with the model Cauchy problem for the twodimensional Laplace equation. As a result, a new analytical method for solving initial-boundary value problems for a two-dimensional heat equation has been developed. The factorization of the transmutation operator is established as a product of two one-dimensional transmutation operators. The form of the transmutation operator establishing the isomorphism of two mathematical models of heat conduction in unbounded media with different physical characteristics was found and descrfibed.
\end{abstract}

\section{Introduction}

The transmutation operator establishes a connection between the solutions of two problems of mathematical physics, one of the problems is considered as a model one.

Example 1. The Cauchy problem for the heat equation [6]

$$
\left\{\begin{array}{c}
\tilde{u}_{t}=\tilde{u}_{x x}, t>0,-\infty<x<\infty \\
\tilde{u}(0, x)=f(x),-\infty<x<\infty
\end{array}\right.
$$

Is model. Let’s consider a new Cauchy problem

$$
\left\{\begin{array}{l}
u_{t}=a^{2} u_{x x}, t>0,-\infty<x<\infty \\
u(0, x)=f(x),-\infty<x<\infty
\end{array}\right.
$$

An operator $J: \tilde{u} \rightarrow u$ that establishes an isomorphism of the solution space for problems (1) and (2) is called a transmutation operator. In this example, the transmutation operator is defined by the formula

$$
u(t, x) \equiv J[\tilde{u}(t, x)]=\tilde{u}\left(a^{2} t, x\right) .
$$

Example 2. Consider the initial-boundary value Cauchy problem for the heat equation

$$
\left\{\begin{array}{l}
u_{1 t}=a_{1}^{2} u_{1 x x}, t>0,-\infty<x<0 \\
u_{1}(0, x)=f_{1}(x),-\infty<x<0
\end{array}\right.
$$

$$
\left\{\begin{array}{l}
u_{2 t}=a_{2}^{2} u_{2 x x}, t>0,0<x<\infty \\
u_{2}(0, x)=f_{2}(x), 0<x<\infty
\end{array}\right.
$$

with internal conjugation conditions

$$
\left\{\begin{aligned}
u_{1}(t, 0) & =u_{2}(t, 0), t>0 \\
\lambda_{1} u_{1 x}(t, 0) & =\lambda_{2} u_{2 x}(t, 0), 0<t
\end{aligned}\right.
$$

Transmutation operator $J_{1}: \tilde{f} \rightarrow f$,

$$
f=f_{1} H(-x)+f_{2} H(x),
$$

where $H(x)$ - Heaviside step function, is defained by formula

$$
f_{2}(x)=\tilde{f}\left(x / a_{2}\right), x>0,
$$

$$
f_{1}(x)=\left(\frac{1}{2}+v\right) \tilde{f}\left(x / a_{1}\right)+\left(\frac{1}{2}-v\right) \tilde{f}\left(-x / a_{1}\right), x<0,
$$

here

$$
v=\frac{1}{2} \frac{\lambda_{2}}{\lambda_{1}} \frac{a_{1}}{a_{2}} .
$$

In the model problem, the initial condition is chosen in the form $\tilde{f}=J_{1}^{-1}[f]$. In more detail, the inverse operator $J_{1}^{-1}$ with respect to the transmutation operator $J_{1}$ has the form 


$$
\begin{aligned}
& \tilde{f}(x)=f_{2}\left(a_{2} x\right), x>0, \\
& \tilde{f}(x)=\frac{2}{1+2 v} f_{1}\left(a_{1} x\right)-\frac{1-2 v}{1+2 v} f_{2}\left(-a_{1} x\right), x<0 .
\end{aligned}
$$

So, the solution to problem (3)-(4) has the form [6]

$$
u(x, t)=\frac{1}{2 \sqrt{\pi t}} \int_{\mathbf{R}} J_{1}\left[\exp \left(-\frac{(x-y)^{2}}{4 t}\right)\right] J_{1}^{-1}[f(y)] d y .
$$

In the authors' work [1,2,21], the formula for solution of problem (3) - (4) is transformed to the form

$$
\begin{aligned}
& u_{1}=\frac{1}{2 a_{1} \sqrt{\pi t}} \int_{-\infty}^{0}\left(e^{-\frac{(x-\xi)^{2}}{4 a_{1}^{2} t}}-e^{-\frac{(x+\xi)^{2}}{4 a_{1}^{2} t}} \frac{1-2 v}{1+2 v}\right) f_{1}(\xi) d \xi+ \\
& +\frac{1}{a_{2} \sqrt{\pi t}} \int_{0}^{\infty} e^{-\frac{\left(a_{1}^{-1} x-a_{2}^{-1} \xi\right)^{2}}{4 t}}(1+2 v)^{-1} f_{2}(\xi) d \xi, t<0, x<0 ;
\end{aligned}
$$$$
u_{2}=\frac{1}{2 a_{1} \sqrt{\pi t}} \int_{-\infty}^{0} e^{-\frac{\left(a_{2}^{-1} x-a_{1}^{-1} \xi\right)^{2}}{4 t}} 2 v(1+2 v)^{-1} f_{1}(\xi) d \xi+(7)
$$$$
+\frac{1}{2 a_{2} \sqrt{\pi t}} \int_{0}^{\infty}\left(e^{-\frac{(x-\xi)^{2}}{4 a_{2}^{2} t}}+e^{-\frac{(x+\xi)^{2}}{4 a_{2}^{2} t}} \frac{1-2 v}{1+2 v}\right) f_{2}(\xi) d \xi, t, x>0 .
$$

Formula (7) is a generalization of the Poisson formula of heat conduction theory [6].

In works $[3,4,5,7,14,16,18,20]$ the theoretical foundations of transmutation operator's method are created. Papers $[8,10-13,15,17,19]$ are devoted to various applications of the method, including the solving of partial differential equations. The most important of the applications of the developed method is the solution of boundary value problems in areas with axial symmetry $[3,11,12]$. The transmutation operator method allows problems with axial symmetry to be reduced to boundary value problems in domains with plane symmetry.

Example 3. Let $\tilde{u}(t, x)$ be the solution of the initialboundary value problem (3) - (4) with parameters $\tilde{a}_{1}, \tilde{a}_{2}, \tilde{\lambda}_{1}, \tilde{\lambda}_{2}$ and initial condition

$$
\tilde{f}(x)=\tilde{f}_{2}(x) H(-x)+\tilde{f}_{2}(x) H(x) .
$$

and $u(t, x)$ is the solution to initial-boundary value problem (3)-(4) with parameters $a_{1}, a_{2}, \lambda_{1}, \lambda_{2}$ and initial condition

$$
f(x)=f_{2}(x) H(-x)+f_{2}(x) H(x),
$$

then the transmutation operator $J$ is defined so that $J[\tilde{f}]=f ; u=J[\tilde{u}]$. The transmutation operator $J$ is a linear combination of stretched or compressed operators and reflections. Let's write out the expression of the transmutation operator $J$

$$
\begin{gathered}
f_{2}(x)=\tilde{f}_{2}\left(\frac{\tilde{a}_{2}}{a_{2}} x\right), x>0 \\
f_{1}(x)=\frac{1+k}{1+\tilde{k}} \tilde{f}_{1}\left(\frac{\tilde{a}_{1}}{a_{2}} x\right)-\frac{\tilde{k}-k}{1+\tilde{k}} \tilde{f}_{2}\left(-\frac{\tilde{a}_{2}}{a_{1}} x\right) .
\end{gathered}
$$

Remark 1. Inverse operator $J^{-1}$ with respect to transmutation operator $J$ can be calculated by formulas

$$
\begin{gathered}
\tilde{f}_{2}(x)=f_{2}\left(\frac{a_{2}}{\tilde{a}_{2}} x\right), x>0, \\
\tilde{f}_{1}(x)=\frac{1+\tilde{k}}{1+k} f_{1}\left(\frac{a_{1}}{\tilde{a}_{2}} x\right)-\frac{k-\tilde{k}}{1+k} f_{2}\left(-\frac{a_{2}}{\tilde{a}_{1}} x\right), x<0,
\end{gathered}
$$

where

$$
k=\frac{\lambda_{2}}{\lambda_{1}} \frac{a_{1}}{a_{2}}, \tilde{k}=\frac{\tilde{\lambda}_{2}}{\tilde{\lambda}_{1}} \frac{\tilde{a}_{1}}{\tilde{a}_{2}} .
$$

The solution to initial-value problem (3)-(4) with parameters $a_{1}, a_{2}, \lambda_{1}, \lambda_{2}$ and initial condition

$$
f(x)=f_{1}(x) H(-x)+f_{2}(x) H(x),
$$

can be obtained by algorithm:

1. find the initial conditions for the model problem according to the rule $\tilde{f}(x)=J^{-1}[f(x)]$;

2. calculate the solution $\tilde{u}(t, x)$ to the model problem;

3. determine the solution of problem (3) - (4) by the formula $u(t, x)=J^{-1}[\tilde{u}(t, x)]$.

\section{Transmutation operator's method for two-dimensional problems}

\subsection{Statement of the problem}

Let us set the goal of obtaining the Poisson formula for solving the heat equation in an unbounded four-layer plate. Consider a separate system of equations describing thermal conductivity in a four-layer medium

$$
u_{i j t}=a_{i}^{2} u_{i j, x x}+b_{j}^{2} u_{i j, y y}, t>0, x, y \in D_{i j},
$$

where

$$
\begin{aligned}
& D_{11}=\{(x, y) / x<0, y<0\}, D_{12}=\{(x, y) / x<0, y>0\}, \\
& D_{21}=\{(x, y) / x>0, y<0\}, D_{22}=\{(x, y) / x>0, y>0\}
\end{aligned}
$$

with initial conditions 


$$
u_{i j}(0, x, y)=f_{i j}(x, y), i, j=1,2
$$

with internal conjugation conditions on coordinate lines $x=0, y=0$

$$
\begin{array}{ll}
u_{1 j}=u_{2 j}, & \lambda_{1} \partial_{x} u_{1 j}=\lambda_{2} \partial_{x} u_{2 j}, x=0 . \\
u_{i 1}=u_{i 2}, & \mu_{1} \partial_{y} u_{i 1}=\mu_{2} \partial_{y} u_{i 2}, y=0 .
\end{array}
$$

Consider the model equation of heat conduction in an unbounded medium

$$
\tilde{u}_{t}=\tilde{u}_{x x}+\tilde{u}_{y y}, t>0, x, y \in R^{2},
$$

with initial condition

$$
\tilde{u}(0, x, y)=\tilde{f}(x, y) .
$$

In order to solve the two-dimensional heat conduction problem (8), (9), (10), it is necessary to determine the transmutation operator. Transmutation operator $J$ establishes isomorphism (8),(9),(10) and (11)-(12), so that $J: \tilde{f} \rightarrow f ; J: \tilde{u} \rightarrow u$.

\subsection{Construction of transmutation operator}

It will be proved here that the transmutation operator $J$ represents as linear combination of stretched or compressed transforms and reflections.

Theorem 1. Transmutation operator $J$ has the form

$$
\begin{gathered}
u_{22}(t, x, y)=\tilde{u}\left(t, x / a_{2}, y / b_{2}\right),(x, y) \in D_{22} \\
u_{12}(t, x, y)=\left(\frac{1}{2}+v\right) \tilde{u}\left(t, x / a_{1}, y / b_{2}\right)+ \\
+\left(\frac{1}{2}-v\right) \tilde{u}\left(t,-x / a_{1}, y / b_{2}\right),(x, y) \in D_{12} \\
\quad u_{21}(t, x, y)=\left(\frac{1}{2}+\mu\right) \tilde{u}\left(t, x / a_{2}, y / b_{1}\right)+ \\
u_{11}(t, x, y)=\left(\frac{1}{2}-\mu\right) \tilde{u}\left(t, x / a_{2},-y / b_{1}\right),(x, y) \in D_{21} \\
+\left(\frac{1}{2}+v\right)\left(\frac{1}{2}-\mu\right) \tilde{u}\left(t, x / a_{1},-y / b_{1}\right)+ \\
+\left(\frac{1}{2}-v\right)\left(\frac{1}{2}+\mu\right) \tilde{u}\left(t,-x / a_{1}, y / b_{1}\right)+ \\
+\left(\frac{1}{2}-v\right)\left(\frac{1}{2}-\mu\right) \tilde{u}\left(t,-x / a_{1},-y / b_{1}\right),(x, y) \in D_{11}
\end{gathered}
$$

here

$$
v=\frac{1}{2} \frac{\lambda_{2}}{\lambda_{1}} \frac{a_{1}}{a_{2}}, \mu=\frac{1}{2} \frac{\mu_{2}}{\mu_{1}} \frac{a_{1}}{a_{2}} .
$$

Proof. Equations (1) are satisfied by construction. Check the conjugation conditions for the component $u_{11}$

$$
\begin{array}{ll}
u_{11}=u_{21}, & \lambda_{1} \partial_{x} u_{11}=\lambda_{2} \partial_{x} u_{21}, x=0, \\
u_{11}=u_{12}, & \mu_{1} \partial_{y} u_{11}=\mu_{2} \partial_{y} u_{12}, y=0 .
\end{array}
$$

Let's check the first of the four conditions. We have

$$
\begin{aligned}
& u_{11}(0, y)=\left(\frac{1}{2}+v\right)\left(\frac{1}{2}+\mu\right) \tilde{u}\left(0, y / b_{1}\right)+ \\
& \quad+\left(\frac{1}{2}+v\right)\left(\frac{1}{2}-\mu\right) \tilde{u}\left(0,-y / b_{1}\right)+ \\
& +\left(\frac{1}{2}-v\right)\left(\frac{1}{2}+\mu\right) \tilde{u}\left(0, y / b_{1}\right)+ \\
& +\left(\frac{1}{2}-v\right)\left(\frac{1}{2}-\mu\right) \tilde{u}\left(0,-y / b_{1}\right)= \\
& =\left(\frac{1}{2}+\mu\right) \tilde{u}\left(0, y / b_{1}\right)+\left(\frac{1}{2}-\mu\right) \tilde{u}\left(0,-y / b_{1}\right) .
\end{aligned}
$$

$$
u_{21}(0, y)=\left(\frac{1}{2}+\mu\right) \tilde{u}\left(0, y / b_{1}\right)+\left(\frac{1}{2}-\mu\right) \tilde{u}\left(0,-y / b_{1}\right) .
$$

The third condition is established similarly. To check the second condition, we calculate the derivatives in the direction of the normal

$$
\begin{gathered}
\lambda_{1} u_{11 x}(x, y)=\left(\frac{1}{2}+v\right)\left(\frac{1}{2}+\mu\right) \frac{\lambda_{1}}{a_{1}} \tilde{u}_{x}\left(0, y / b_{1}\right)+ \\
+\left(\frac{1}{2}+v\right)\left(\frac{1}{2}-\mu\right) \frac{\lambda_{1}}{a_{1}} \tilde{u}_{x}\left(0,-y / b_{1}\right)- \\
\quad-\left(\frac{1}{2}-v\right)\left(\frac{1}{2}+\mu\right) \frac{\lambda_{1}}{a_{1}} \tilde{u}_{x}\left(0, y / b_{1}\right)- \\
-\left(\frac{1}{2}-v\right)\left(\frac{1}{2}-\mu\right) \frac{\lambda_{1}}{a_{1}} \tilde{u}_{x}\left(0,-y / b_{1}\right)= \\
=2 v\left(\frac{1}{2}+\mu\right) \frac{\lambda_{1}}{a_{1}} \tilde{u}_{x}\left(0, y / b_{1}\right)+2 v\left(\frac{1}{2}-\mu\right) \frac{\lambda_{1}}{a_{1}} \tilde{u}_{x}\left(0,-y / b_{1}\right) .
\end{gathered}
$$

Then

$$
\begin{aligned}
& \lambda_{2} u_{21 x}(0, y)=\left(\frac{1}{2}+\mu\right) \frac{\lambda_{2}}{a_{2}} \tilde{u}\left(0, y / b_{1}\right)+ \\
& +\left(\frac{1}{2}-\mu\right) \frac{\lambda_{2}}{a_{2}} \tilde{u}\left(x / a_{2},-y / b_{1}\right) .
\end{aligned}
$$

Further, the fourth conjugation condition is proved similarly. The conjugation conditions for the functions $u_{12}, u_{21} u_{22}$. 
Theorem 2. The inverse operator $J^{-1}$ to the transfmutation operator $J$ has the form

$$
\begin{gathered}
\tilde{u}(x, y)=u_{22}\left(a_{2} x, b_{2} y\right),(x, y) \in D_{22}, \\
\tilde{u}(x, y)=\frac{2}{1+2 v} u_{12}\left(a_{1} x, b_{2} y\right)-\frac{1-2 v}{1+2 v} u_{22}\left(-a_{1} x, b_{2} y\right), \\
(x, y) \in D_{12}, \\
\tilde{u}(x, y)=\frac{2}{1+2 \mu} u_{21}\left(a_{2} x, b_{1} y\right)-\frac{1-2 \mu}{1+2 \mu} u_{22}\left(a_{2} x,-b_{1} y\right), \\
\tilde{u}(x, y)=\frac{4}{(1+2 v)(1+2 \mu)} u_{11}\left(a_{1} x, b_{1} y\right)- \\
-\frac{1-2 \mu}{1+2 \mu}\left(\frac{2}{1+2 v} u_{12}\left(a_{1} x,-b_{2} y\right)-\frac{1-2 v}{1+2 v} u_{22}\left(-a_{1} x,-b_{2} y\right)\right) \\
-\frac{1-2 v}{1+2 v}\left(\frac{2}{1+2 \mu} u_{21}\left(-a_{2} x, b_{1} y\right)-\frac{1-2 \mu}{1+2 \mu} u_{22}\left(-a_{2} x,-b_{1} y\right)\right)- \\
-\frac{1-2 \mu}{1+2 \mu} \frac{1-2 v}{1+2 v} u_{22}\left(-a_{2} x,-b_{2} y\right) \tilde{u}(-x,-y),(x, y) \in D_{11} .
\end{gathered}
$$

Proof. From the first equation of (13) we determine the function $\tilde{u}(x, y)$ in the domain $D_{22}$. Then we move on to the second equation. Considering the first step, we get the expression of function $\tilde{u}(x, y)$ in the area $D_{12}$. We finish the calculations by considering the fourth equation from (13), using the previously obtained values of the function $\tilde{u}(x, y)$ in the domains $D_{22}, D_{21}, D_{12}$.

In the next section, for a better understanding of the construction of the transfmutation operator, we will establish the possibility of its decomposition as a product of simple transfmutation operators $J_{1}$ and $J_{2}$.

\subsection{Factorization of the transmutation operator}

The transfmutation operator $J_{1}$ acts on a function of the variable $x$. Similarly, we define a transformation operator $J_{2}$ that acts on a function of the variable y. Let a function $\tilde{h}(y)$ be continuously differentiable on the real axis, and let the function

$$
h(y)=h_{1}(y) H(-y)+h_{2}(y) H(y)
$$

was defined as follow

$$
\begin{aligned}
& h_{2}(y)=\tilde{h}\left(y / b_{2}\right), y>0, \\
& h_{1}(y)=\left(\frac{1}{2}+\mu\right) \tilde{h}\left(y / b_{1}\right)+\left(\frac{1}{2}-\mu\right) \tilde{h}\left(-y / b_{1}\right), y<0
\end{aligned}
$$

where

$$
\mu=\frac{1}{2} \frac{\mu_{2}}{\mu_{1}} \frac{b_{1}}{b_{2}},
$$

then the function

$$
h(y)=h_{1}(y) H(-x)+h_{2}(y) H(x) \text { is continuously }
$$

differentiable in $R \backslash\{0\}$ and the conjugation condition

$$
\begin{aligned}
& h_{1}(0)=h_{2}(0), \\
& \mu_{1} h_{1 y}(0)=\mu_{2} h_{2 y}(0)
\end{aligned}
$$

are hold. So, for transmutation oherator $J_{2}$ the following formula is true:

$$
h(y)=J_{2}[\tilde{h}(y)] \text {. }
$$

Inverse operator $J_{2}^{-1}$ takes form

$$
\begin{aligned}
& \tilde{h}(y)=h_{2}\left(b_{2} y\right), y>0, \\
& \tilde{h}(x)=\frac{2}{1+2 v} h_{1}\left(b_{1} y\right)-\frac{1-2 v}{1+2 v} h_{2}\left(-b_{1} y\right), y<0
\end{aligned}
$$

Theorem 3. The transmutation operator $J$ is factorized as the product of an operator $J_{1}$ that acts on the variable $\mathrm{x}$ and an operator $J_{2}$ that acts on $\mathrm{y}$, i.e. $J=J_{1} J_{2}$. The inverse operator $J^{-1}$ to the transmutation operator also admits factorization in the form $J^{-1}=J_{2}^{-1} J_{1}^{-1}$.

The proof follows from the definition of the operators $J_{1}$ and $J_{2}$.

Theorem 4. The transmutation operator $J$ and the Laplace operator $\Delta$ are commutative

$$
\begin{gathered}
\Delta=\left(a_{1}^{2} \partial_{x x}+b_{1}^{2} \partial_{y y}\right) H(-x) H(-y)+ \\
\left(a_{2}^{2} \partial_{x x}+b_{1}^{2} \partial_{y y}\right) H(x) H(-y)+ \\
+\left(a_{1}^{2} \partial_{x x}+b_{2}^{2} \partial_{y y}\right) H(-x) H(y)+ \\
\left(a_{2}^{2} \partial_{x x}+b_{2}^{2} \partial_{y y}\right) H(-x) H(-y),
\end{gathered}
$$

i.e. $J \Delta=\Delta J$.

The proof follows from Theorem 1 .

\subsection{Extending the Results to the Vector Case}

Let a vector-function

$$
f(x)=f_{1}(x) H(-x)+f_{2}(x) H(x),
$$

where

$$
f_{i}(x)=\left(\begin{array}{lll}
f_{i 1}(x) & \ldots & \left.f_{\text {in }}(x)\right)^{*}, i=1,2
\end{array}\right.
$$

be continuous in, continuously differentiable in $R$, and continuously differentiable in $R \backslash\{0\}$. The parameters 
$a_{1}, a_{2}, \lambda_{1}, \lambda_{2}$ are matrices of $n \times n$ size for each of which all eigenvalues are different and positive.

The definition of a matrix argument function can be found in the book [9]. We present an unknown definition of the vector function of a matrix argument.

Definition 1. Suppose, that all eigenvalues of matrices $A$ and $B$ of dimensions $n \times n$ are different and positive, and the vector -function $f(x)$ is given in $R$ and has $n \times 1$-size, then the vector -function $f(A x)$ of the matrix argument is determined by the rule

$$
f(A x)=\sum_{k=1}^{n} f_{k}(A x) e_{k}, \quad e_{k}=\left(\begin{array}{lllll}
0 & \ldots & 1 & \ldots & 0
\end{array}\right)^{*} .
$$

and the vector-function $B f(A x)$ is defined by the rule

$$
B f(A x)=B f(x)(x \rightarrow A x)
$$

Let us explain the last formula. First, you need to calculate the product $B f(x)$, and then to apply the definition of a vector- function of a matrix argument. However, the order of operations cannot be changed.

Example 4. If we have matrices

$$
A=\left(\begin{array}{ll}
-1 & 3 \\
-2 & 4
\end{array}\right), B=\left(\begin{array}{ll}
1 & 2 \\
0 & 3
\end{array}\right)
$$

then

$$
\begin{gathered}
B f(A x)=\left(\begin{array}{c}
-5 x+29 x^{2} \\
-6 x+30 x^{2}
\end{array}\right), \\
B f(x)(x \rightarrow A x)=\left(\begin{array}{c}
-x+17 x^{2} \\
-2 x+18 x^{2}
\end{array}\right),
\end{gathered}
$$

and

$$
B f(A x) \neq B f(x)(x \rightarrow A x)
$$

Note, if matrices $A$ and $B$ are permute the equality is true.

The vector analogs of transformation operators have the following form:

Transmutation operator $J_{1}: \tilde{f} \rightarrow f$, where

$$
f=f_{1} H(-x)+f_{2} H(x),
$$

$H(x)$ - Heaviside step function, and $f$ is defined by equality

$$
\begin{gathered}
f_{2}(x)=\tilde{f}\left(a_{2}^{-1} x\right), x>0, \\
f_{1}(x)=\left(\left(\frac{\mathrm{E}}{2}+v\right) \tilde{f}(x)+\left(\frac{\mathrm{E}}{2}-v\right) \tilde{f}(-x)\right)\left(x \rightarrow a_{1}^{-1}\right), x<0,
\end{gathered}
$$

where

$$
v=\frac{1}{2} a_{1} \lambda_{1}^{-1} \lambda_{2} a_{2}^{-1}
$$

The inverse vector transmutation operator $J_{1}^{-1}$ for the operator $J_{1}$ has the form

$$
\tilde{f}(x)=f_{2}\left(a_{2} x\right), x>0,
$$

$$
\begin{aligned}
& \tilde{f}(x)=\left(2(\mathrm{E}+2 v)^{-1} f_{1}(x)-(\mathrm{E}-2 v)(\mathrm{E}+2 v)^{-1} f_{2}(-x)\right) \\
& \left(x \rightarrow a_{1} x\right), x<0 .
\end{aligned}
$$

The vector analogue of the initial-boundary value Cauchy problem (3) - (4) for the heat equation is posed as follows: find a solution to the system of differential equations

$$
\begin{aligned}
& \left\{\begin{array}{l}
u_{1 t}=a_{1}^{2} u_{1 x x}, t>0,-\infty<x<0, \\
u_{1}(0, x)=f_{1}(x),-\infty<x<0,
\end{array}\right. \\
& \left\{\begin{array}{l}
u_{2 t}=a_{2}^{2} u_{2 x x}, t>0,0<x<\infty, \\
u_{2}(0, x)=f_{2}(x), 0<x<\infty,
\end{array}\right.
\end{aligned}
$$

satisfied the conditions of internal conjugation

$$
\left\{\begin{aligned}
u_{1}(t, 0) & =u_{2}(t, 0), t>0, \\
\lambda_{1} u_{1 x}(t, 0) & =\lambda_{2} u_{2 x}(t, 0), 0<t,
\end{aligned}\right.
$$

where matrices $\lambda_{1}, \lambda_{2} n \times n$ - size eigenvalues of wich are different and positive. The solution of this vector analogue of problem (3) - (4) can be determined by the formula with help of transmutation operator $J_{1}$ and its inverse operator $J_{1}^{-1}$ :

$$
u(x, t)=\frac{1}{2 \sqrt{\pi t}} \int_{\mathbf{R}} J_{1}\left[\exp \left(-\frac{(x-y)^{2}}{4 t}\right)\right] J_{1}^{-1}[f(y)] d y
$$

\section{Solution of the initial-boundary value problem for the heat equation in an unbounded piecewise-homogeneous plate}

We find the solution to the initial-boundary value problem (1) - (3) using Theorem 1. We apply the transmutation operator $J$ from theorem 1 to problem (1) - (3). In the images, we obtain problem (4) - (5), in which the initial state of the temperature field is determined by the formula $\tilde{f}=J[f]$. We use the Poisson formula for the two-dimensional Poisson equation

$$
\tilde{u}(t, x, y)=\frac{1}{2 \pi t} \int_{R^{2}} e^{-\frac{(x-\xi)^{2}+(y-\eta) 2}{4 t}} \tilde{f}(\xi, \eta) d \xi d \eta
$$

On the base of theorem 1 we have 


$$
u(t, x, y)=\frac{1}{2 \pi t} \int_{R^{2}} J\left[e^{-\frac{(x-\xi)^{2}+(y-\eta)^{2}}{4 t}}\right] \tilde{f}(\xi, \eta) d \xi d \eta .
$$

In each of the four areas $D_{22}, D_{21}, D_{12}, D_{11}$, we obtain a solution to problem (1) - (3)

$$
\begin{aligned}
& u_{22}(t, x, y)=\frac{1}{2 \pi t} \int_{R^{2}} e^{-\frac{\left(x / a_{2}-\xi\right)^{2}+\left(y / b_{2}-\eta\right) 2}{4 t}} \tilde{f}(\xi, \eta) d \xi d \eta, \\
& (x, y) \in D_{22}, \\
& u_{12}(t, x, y)=\frac{1}{2 \pi t} \int_{R^{2}}\left(\left(\frac{1}{2}+v\right) e^{-\frac{\left(x / a_{1}-\xi\right)^{2}+\left(y / b_{2}-\eta\right) 2}{4 t}}+\right. \\
& \left.+\left(\frac{1}{2}-v\right) e^{-\frac{\left(x / a_{1}+\xi\right)^{2}+\left(y / b_{2}-\eta\right) 2}{4 t}}\right) \tilde{f}(\xi, \eta) d \xi d \eta,(x, y) \in D_{12}, \\
& u_{21}(t, x, y)=\frac{1}{2 \pi t} \int_{R^{2}}\left(\left(\frac{1}{2}+\mu\right) e^{-\frac{\left(x / a_{2}-\xi\right)^{2}+\left(y / b_{1}-\eta\right) 2}{4 t}}+\right. \\
& \left.+\left(\frac{1}{2}-\mu\right) e^{-\frac{\left(x / a_{2}-\xi\right)^{2}+\left(y / b_{1}+\eta\right) 2}{4 t}}\right) \tilde{f}(\xi, \eta) d \xi d \eta,(x, y) D_{21}, \\
& u_{11}(t, x, y)=\frac{1}{2 \pi t} \int_{R^{2}}\left(\left(\frac{1}{2}+v\right)\left(\frac{1}{2}+\mu\right) e^{-\frac{\left(x / a_{1}-\xi\right)^{2}+\left(y / b_{1}-\eta\right) 2}{4 t}}+\right. \\
& +\left(\frac{1}{2}+v\right)\left(\frac{1}{2}-\mu\right) e^{-\frac{\left(x / a_{1}-\xi\right)^{2}+\left(y / b_{1}+\eta\right) 2}{4 t}}+ \\
& \left.+\left(\frac{1}{2}-v\right)\left(\frac{1}{2}-\mu\right) e^{-\frac{\left(x / a_{1}+\xi\right)^{2}+\left(y / b_{1}+\eta\right)^{2}}{4 t}}\right) \tilde{f}(\xi, \eta) d \xi d \eta, i n D_{11} . \\
& +\left(\frac{1}{2}+\mu\right) e^{-\frac{\left(x / a_{1}+\xi\right)^{2}+\left(y / b_{1}-\eta\right) 2}{4 t}}+
\end{aligned}
$$

Further, we can simplify the found formulas. To do this, we use the expression of the function $\tilde{f}(\xi, \eta)$ from theorem 2. As a result of simplifications for each of the four components, we obtain formulas in which only the initial data (9) participate. For example, we present the formula for the component $u_{11}$

$$
\begin{gathered}
u_{11}=\frac{1}{4 \pi a_{1} b_{1} t} \int_{D_{11}}\left(e^{-\frac{(x-\xi)^{2}}{4 a_{1}^{2} t}}-e^{-\frac{(x+\xi)^{2}}{4 a_{1}^{2} t}} \frac{1-2 v}{1+2 v}\right) \\
\left(e^{-\frac{(y-\eta)^{2}}{4 b_{1}^{2} t}}-e^{-\frac{(y+\eta)^{2}}{4 b_{1}^{2} t}} \frac{1-2 \mu}{1+2 \mu}\right) f_{11}(\xi, \eta) d \xi d \eta+
\end{gathered}
$$

$$
+\frac{1}{2 a_{1} b_{2} \pi t} \int_{D_{12}}\left(e^{-\frac{\left(x / a_{1}-\xi / a_{1}\right)^{2}}{4 t}}-e^{-\frac{\left(x / a_{1}+\xi / a_{1}\right)^{2}}{4 t}} \frac{1-2 v}{1+2 v}\right) .
$$$$
\cdot(1+2 \mu)^{-1} e^{-\frac{\left(y / b_{1}-\eta / b_{2}\right)^{2}}{4 t}} d \eta f_{12}(\xi, \eta) d \xi d \eta+
$$

$$
\begin{gathered}
+\frac{1}{2 a_{2} b_{1} \pi t} \int_{D_{21}} e^{-\frac{\left(x / a_{1}-\xi / b_{1}\right)^{2}}{4 t}}(1+2 v)^{-1} . \\
\left.\cdot e^{-\frac{\left(y / b_{1}-\eta / b_{1}\right)^{2}}{4 t}}-e^{-\frac{\left(y / b_{1}+\eta / b_{1}\right)^{2}}{4 t}} \frac{1-2 \mu}{1+2 \mu}\right) . \\
\cdot f_{21}(\xi, \eta) d \xi d \eta+ \\
+\frac{1}{\pi a_{2} b_{2} t} \int_{D_{22}} e^{-\frac{\left(x / a_{1}-\xi / a_{2}\right)^{2}}{4 t}} e^{-\frac{\left(y / b_{1}-\eta / b_{2}\right)^{2}}{4 t}} . \\
(1+2 v)^{-1}(1+2 \mu)^{-1} f_{22}(\xi, \eta) d \xi d \eta, x, y<0 .
\end{gathered}
$$

Similarly, the components $u_{12}, u_{21}, u_{22}$ can be found.

\section{Discussion}

The method of Fourier integral transforms, well-known in classical mathematics, is an alternative to the method of transmutation operators developed in this article. Let's compare both methods. Let us associate problem (9) (11) with two one-dimensional Sturm-Liouville problems. The first problem is to determine a bounded on the real axis solution to the equation with piecewise constant coefficients

$$
\begin{aligned}
& a_{1}^{2} \varphi_{1 x x}+\alpha^{2} \varphi_{1}, x<0, \\
& a_{2}^{2} \varphi_{2 x x}+\alpha^{2} \varphi_{2}, x>0
\end{aligned}
$$

with internal conjgation conditions on the stright line $x=0$

$$
\varphi_{1}=\varphi_{2}, \quad \lambda_{1} \varphi_{1 x}=\lambda_{2} \varphi_{2 x}, x=0 .
$$

The solution to problem (16) - (17) defines the kernel of the inverse Fourier integral transform $F_{1 x}^{-1}$ with a point of division on the real axis [21]

$$
f(x) \equiv F_{1 x}^{-1}[\tilde{f}(\alpha)]=\int_{-\infty}^{\infty} \varphi(x, \alpha) \tilde{f}(\alpha) d \alpha .
$$

Then consider the dual Sturm-Liouville problem: find a bounded on the real axis solution of the equation with piecewise constant coefficients

$$
\begin{aligned}
& a_{1}^{2} \varphi_{1 x x}^{*}+\alpha^{2} \varphi_{1}^{*}=0, x<0, \\
& a_{2}^{2} \varphi_{2 x x}^{*}+\alpha^{2} \varphi^{*}{ }_{2}=0, x>0
\end{aligned}
$$


with internal conjgation conditions on the stright $x=0$

$$
\lambda_{2} \varphi_{1}^{*}=\lambda_{1} \varphi_{2}^{*}, \quad \varphi_{1 x}^{*}=\varphi_{2 x}^{*}, x=0
$$

The solution to this dual problem (18) - (19) defines the kernel of the direct Fourier integral transform $F_{1 x}$ with a point of division on the real axis

$$
\tilde{f}(\alpha) \equiv F_{1 x}[f(x)]=\int_{-\infty}^{\infty} \varphi^{*}(x, \alpha) f(x) d x .
$$

The expressions for the kernels of integral transforms are written out in [21].

The direct $F_{1 y}$ and inverse $F_{1 y}^{-1}$ Fourier transforms with the division point in the variable $\mathrm{y}$ are defined similarly. Moreover, in the Sturm-Liouville problem, it is necessary to replace $\alpha \rightarrow \beta, a_{i} \rightarrow b_{i} ; \lambda_{i} \rightarrow \mu_{i}, i=1,2$.

To solve problem (9) - (11), we apply the Fourier integral transforms $F_{1 x}$ and $F_{1 y}$. According to the scheme proposed in [21], we obtain a formula for solving problem (9) - (11):

$$
u(t, x, y)=F_{1 x}^{-1} F_{1 y}^{-1}\left[e^{-\left(\alpha^{2}+\beta^{2}\right) t} F_{1 x} F_{1 y}(f(x, y))\right] .
$$

Comparing formulas (15) and (20), we conclude the advantage of transmutation operator's method. In formula (15), the solution is given by a double integral, and in formula (20) it is need to use four -times integral. Thus, the transmutation operator's method has all the advantages of the integral transforms method and is more efficient from a computational point of view.

\section{Conclusion}

The article has solves a number of problems. In particular, a solution is found for a two-dimensional problem of heat conduction in an unbounded thin plate with four sections with different thermophysical parameters. The solution is found in terms of the transmutation operator. The construction of the transmutation operator and its inverse is found, the factorization of the transmutation operator is found in the form of the product of transmutation operators with respect to the variables $\mathrm{x}$ and $\mathrm{y}$. The connection between the transmutation operator method and the method of integral transforms is established. It is shown that from the computational point of view the method of transmutation operators is more efficient than the method of integral transforms.

The method of transmutation operators makes it possible to predict the structure of the temperature field of an unbounded piecewise homogeneous thin plate from the known temperature field of a model piecewise homogeneous (or homogeneous) thin plate.

Just like the method of integral transforms, the method of transmutation operators is universal. It can be used to solve differential equations of hyperbolic and elliptic types. The technique of applying the method is similar to the method of Fourier integral transforms:

1.From the problem in the originals, we pass to the problems in the images,

2. we solve the problem in images,

3 . back to the originals space.

The difference lies in the fact that the image space coincides with the space of the originals of a certain model problem.

Example 4. Consider the initial-boundary value Cauchy problem for the equation of vibrations of a infinite string with two sections of different dencity

$$
\begin{gathered}
\left\{\begin{array}{l}
u_{1 t t}=a_{1}^{2} u_{1 x x}, t>0,-\infty<x<0, \\
u_{1}(0, x)=f_{1}(x),-\infty<x<0, \\
\partial_{t} u_{1}(0, x)=0,-\infty<x<0,
\end{array}\right. \\
\left\{\begin{array}{l}
u_{2 t t}=a_{2}^{2} u_{2 x x}, t>0,0<x<\infty \\
u_{2}(0, x)=f_{2}(x), 0<x<\infty \\
\partial_{t} u_{2}(0, x)=0,0<x<\infty
\end{array}\right.
\end{gathered}
$$

with internal conjgation conditions

$$
\left\{\begin{array}{c}
u_{1}(t, 0)=u_{2}(t, 0), t>0, \\
\lambda_{1} u_{1 x}(t, 0)=\lambda_{2} u_{2 x}(t, 0), 0<t .
\end{array}\right.
$$

The Cauchy problem for the equation of string vibrations is a model problem

$$
\left\{\begin{array}{l}
\tilde{u}_{t t}=\tilde{u}_{x x}, t>0,-\infty<x<\infty, \\
\tilde{u}(0, x)=\tilde{f}(x),-\infty<x<0, \\
\partial_{t} \tilde{u}(0, x)=0,-\infty<x<\infty,
\end{array}\right.
$$

The solution to the model problem is determined by the d'Alembert formula

$$
\tilde{u}(t, x)=\frac{\tilde{f}(x-t)+\tilde{f}(x+t)}{2} .
$$

Thus, the solution to problem (21) - (22) has the form

$$
u(t, x)=J_{1 x}\left[\frac{\tilde{f}(x-t)+\tilde{f}(x+t)}{2}\right] \text {. }
$$

In the expanded form, the aforeentioned formula is given in the work [18].

The work is supported by the Scientific Research by Educational Organizations in 2020-2022 Project under Grant No FSFS- 2020-0031 (075-03-2020-079/2).

\section{References}

1. I.I. Bavrin, O.E. Yaremko, Differ. Equ., 40(8), 1149-1160 (2004) https://doi.org/10.1023/B:DIEQ.0000049832.37277. 95 
2. I.I. Bavrin, O.E. Yaremko, Doklady Mathematics, 68(3), 371-375 (2003)

3. R. Carroll, Transmutation and Operator Differential Equations (North Holland, 1979)

4. R. Carroll, Transmutation, Scattering Theory and Special Functions (North-Holland, 1982)

5. R. Carroll, Transmutation theory and applications (North-Holland, 1985)

6. R. Courant, D. Hilbert, Methods of Mathematical Physics, vol II (Wiley) New York, 1962)

7. M.M. Dzrbashian, Harmonic Analysis and Boundary Value Problems in the Complex Domain (Birkhauser, 1993)

8. A. Fitouhi, I. Jebabli, E.L. Shishkina, S.M. Sitnik, EJDE, 130, 1-27 (2018)

9. F.R. Gantmacher. The Theory of Matrices (Chelsea, New York, 1959)

10. V.V. Katrakhov, S.M. Sitnik, Doklades of the Russian Academy of Sciences, 337(3), 307-311, (1994)

11. V.V. Kravchenko, S.M. Torba, CAOT, 9(2), 379429 (2015)

12. V.V. Kravchenko, V.A. Vicente-Benítez, Math. Meth. Appl. Sci., 43(16), 9455-9486 (2020)

13. A.A. Kilbas, H.M. Srivastava, J.J. Truhillo, Theory and Applicationsof Fractional Differential Equations (Elsevier, 2006)

14. A.A. Kilbas, M. Saigo, $H$ - transforms. Theory and applications (Chapman and Hall, CRC.2004)

15. N.H. Mahmoud, Transactions of the AMS, 352(8), 3687-3706 (2000)

16. S.G. Samko, A.A. Kilbas, O.I. Marichev, Integrals and Derivatives of Fractional Order and Some Their Applications (Nauka i Tekhnika, Minsk, 1987) [in Russian]

17. E. Shishkina, S. Sitnik, Transmutations, Singular and Fractional Differential Equations with Applications to Mathematical Physics, (Elsevier, 2020)

18. S.M Sitnik, O. Yaremko, N. Yaremko, Transmutation Operators and Applications (Springer 2020)

19. Ta Li, Proc. AMS, 11(2), 290-298 (1960)

20. B.M. Levitan, The theory of generalized shift operators (Nauka, Moscow, 1973)

21. O.E. Yaremko, JMAG, 9(4), 594-603 (2013) 